\title{
RELIABILITY OF THE ESTIMATION OF
}

\section{TOTAL HEPATIC BLOOD FLOW BY DOPPLER ULTRASOUND}

\section{IN PATIENTS WITH CIRRHOTIC PORTAL HYPERTENSION}

Annalisa Berzigotti ${ }^{1,2,3}$, Enric Reverter ${ }^{1,2}$, Ángeles García-Criado ${ }^{3}$, Juan G. Abraldes ${ }^{1,2}$, Federica Cerini ${ }^{1,2}$, Juan Carlos García-Pagán ${ }^{1,2}$, Jaime Bosch ${ }^{1,2}$

${ }^{1}$ Hepatic Hemodynamic Laboratory, Liver Unit, Hospital Clinic, University of Barcelona, Spain, ${ }^{2}$ Centro de Investigación Biomédica en Red de Enfermedades

Hepáticas y Digestivas (Ciberehd), Spain, ${ }^{3}$ Centre de Diagnostic per l'Imatge, Hospital Clinic, Barcelona, Spain

Correspondence: Dr. Jaime Bosch, Hepatic Hemodynamic Laboratory, Liver Unit, Hospital Clínic, Villarroel 170, 08036 Barcelona, Spain. Phone: +34 932275400 (5790); E-mail address: jbosch@clinic.ub.es

\section{Short title: Hepatic blood flow in cirrhosis}

Word count: 3095

This work is supported in part by grants from Instituto de Salud Carlos III (PS09/01261). CIBERehd is funded by Instituto de Salud Carlos III. 


\section{ABSTRACT}

Background \& Aims. Hepatic blood flow (HBF) is best estimated by Fick's method during indocyanine green constant infusion (ICG-HBF) on hepatic vein catheterization. We investigated the consistency and agreement of HBF measured by Doppler ultrasound (US-HBF) as compared with ICG-HBF in portal hypertensive patients with cirrhosis.

Methods. In 50 patients observed for HVPG measurement (56\% compensated; Child

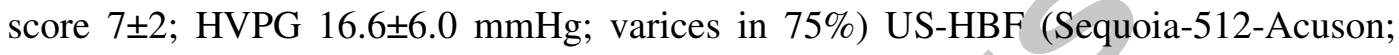
4.5-7 $\mathrm{MHz}$ convex probe; $\mathrm{US}-\mathrm{HBF}=$ hepatic artery blood flow + portal vein blood flow) and ICG-HBF (Fick's method after an equilibration period of at least 45 minutes of ICG bolus of $5 \mathrm{mg}+$ constant rate infusion of $0.2 \mathrm{mg} / \mathrm{min}$ ). Intraclass correlation coefficient (ICC) for consistency and absolute agreement between US-HBF and ICG-HBF were calculated.

Results. Mean ICG-HBF and US-HBF were similar, being respectively $1004 \pm 543$ $\mathrm{ml} / \mathrm{min}$, and $994 \pm 494 \mathrm{ml} / \mathrm{min}$ ( $\mathrm{p}=0.661$ vs. ICG-HBF). However, results in individual patients disclosed marked differences between the two methods $(386 \pm 415 \mathrm{ml} / \mathrm{min})$, and showed only moderate consistency (ICC 0.456; $\mathrm{p}<0.0001$ ), absolute agreement (ICC 0.461; $\mathrm{p}<0.0001)$ and linear correlation $(\mathrm{R}=0.464 ; \mathrm{p}<0.0001)$. The discrepancy between the two methods was maximal in patients with poor liver function, high HBF by any technique and more arterialized liver circulation. Hepatic artery blood flow $\geq 40 \%$ of US-HBF indicated with $90 \%$ specificity a discrepancy $\geq 20 \%$ between US-HBF and ICG-HBF.

Conclusions: HBF estimations by Doppler-ultrasound and ICG are significantly correlated, but their discrepancy in individual cases is high. Estimation of HBF by 
Doppler-US should be considered unreliable in patients with poor hepatic function and large liver arterialization.

Abstract word count: 250

Keywords. Non-invasive methods. Portal hypertension. Hepatic veins. HVPG. 


\section{INTRODUCTION}

In patients with cirrhosis and portal hypertension the goal of therapy is to reduce portal pressure without deteriorating hepatic perfusion [1]. While hepatic venous pressure gradient (HVPG) measurement is a consistent and reproducible surrogate of portal pressure in cirrhosis [2], there is an unmet need for reliable techniques to assess total hepatic blood flow (HBF) in clinical practice.

The indocyanine green (ICG) constant infusion technique [3] has been widely used to estimate HBF by Fick's method in healthy subjects [4] and in patients with cirrhosis [5, 6]. This method is objective and reproducible, and is currently considered the gold standard for HBF quantitative measurement. However, this technique requires hepatic vein catheterization, and cannot be used routinely.

Given the limitations of ICG-based HBF measurement (ICG-HBF), non-invasive methods to estimate $\mathrm{HBF}$ have been investigated. Doppler duplex ultrasonography (DUS) allows a non-invasive study of abdominal organs and abdominal circulation in real time, and has been widely used to assess the circulatory abnormalities occurring in patients with cirrhosis and portal hypertension [7, 8]. DUS allows evaluating separately the two components of total HBF, namely portal vein blood flow (PBF) and hepatic artery blood flow (HABF) [9], but while DUS has been proved reliable for PBF estimation [10], very limited and inconclusive data exist on DUS-based measurement of total HBF in patients with cirrhosis [11].

The aim of this study was to assess the consistency and agreement of Doppler ultrasound for the assessment of hepatic blood flow in patients with cirrhosis by comparing this method with HBF estimated by the gold standard (HBF by ICG by Fick's method during hepatic vein catheterization). 


\section{METHODS}

This study was approved by the Ethics Committee of Hospital Clinic. The nature of the study was explained to the patients, and a written informed consent was obtained in each case, according to the principles of the Declaration of Helsinki (revision of Edinburgh 2000).

\section{Patients}

50 patients with liver cirrhosis and hepatopetal portal blood flow, with valid measurements of ICG-HBF and valid measurements of both PBF and HABF by DUS, admitted to our Laboratory for hepatic venous pressure gradient measurement, were included in this study. Exclusion criteria were the following: age $<18$ or $>80$ years; pregnancy; hepatocellular carcinoma; portal vein thrombosis; extraction index of ICG < 0.1 [5]; insufficient visualization of the portal vein and hepatic artery. This last criterion led to the exclusion of 9 patients.

Tab. 1 shows the main clinical and laboratory characteristics of the studied population.

Hepatic venous pressure gradient and HBF by Indocyanine green (ICG-HBF) measurement

Patients underwent hepatic vein catheterisation in the morning after at least 8 hour fasting. Under local anaesthesia, with ultrasonographic guidance (SonoSite Inc, Bothell, WA) a 8F venous catheter introducer (Axcess; Maxxim Medical, Athens, TX, USA) was placed in the right internal jugular vein using the Seldinger technique. Thereafter, a 7F balloon-tipped catheter (Edwards Lifesciences, Irvine, CA, USA) was advanced into 
the right hepatic vein to measure wedged and free hepatic venous pressures (WHVP and FHVP, respectively) by the connection to external electro-mechanical transducer and polygraph (Mac-Lab®, GE Healthcare, Freiburg, Germany). HVPG was calculated as WHVP -FHVP [2].

Preceded by a priming dose of $5 \mathrm{mg}$, a solution of indocyanine green (Pulsion Medical Systems, Munich, Germany) was infused intravenously at a constant rate of $0.2 \mathrm{mg} / \mathrm{min}$. After an equilibration period of at least 40 minutes to achieve a steady-state, 4 separate sets of simultaneous samples of peripheral and hepatic venous blood were obtained for the measurement of hepatic blood flow according to the Fick's method as previously described [12]. To avoid interferences from differences in plasma turbidity, the Nielsen's correction was used [5] at the moment of reading ICG concentration in the samples by spectrophotometry (SP-830, Turner Biosystems, Sunnyvale, CA, USA).

Briefly, ICG clearance was calculated as ICG constant infusion velocity/mean concentration of ICG in the peripheral venous blood. ICG extraction index was calculated as: (concentration of ICG in the peripheral venous blood - concentration of ICG in the hepatic venous blood)/concentration of ICG in peripheral venous blood. Hepatic plasma flow was estimated as ICG clearance/ICG extraction index. Finally, hepatic blood flow was estimated as: hepatic plasma flow /(1- hematocrit).

\section{HBF by Doppler Ultrasound (US-HBF)}

Patients underwent DUS examination on the same morning of hepatic vein catheterization, after an overnight fast, before undergoing the invasive procedure. They were invited to lie supine for 10 minutes. Thereafter, Doppler measurements were 
performed using a Siemens ACUSON Sequoia ${ }^{\mathrm{TM}} 512$ (Acuson, Mountain View, CA, USA) ultrasound system, by the same physician in order to avoid interobserver variability. A 3.5-5 MHz convex probe provided by a color, power and pulsed Doppler software was used. Following current recommendations $[7,8]$, portal vein and hepatic artery were imaged by B-mode. The gain was reduced and the image size made as large as possible to improve resolution. Diameter and flow velocity were measured in both vessels during short time suspended normal respiration using an oblique scan in the epigastrium in a standardized site (crossing of hepatic artery and portal vein). Insonation angles of $50-55^{\circ}$ were used for these measurements. The Doppler sample was positioned in the center of the lumen, setting its dimension as wide as $\geq 50 \%$ of the vessel diameter. Measurements were taken in triplicate, and the results were expressed as the mean value. Variability between different measures was $<10 \%$. Intraobserver variability was previously assessed and was $<10 \%$.

Time averaged maximum velocity in the portal vein and in the hepatic artery was obtained from delineation of the Doppler spectral signal. Portal blood velocity was calculated as time averaged maximum velocity multiplied by 0.57 , assuming the portal velocity profile as parabolic, as previously reported [13, 14]. Similarly, hepatic artery velocity was calculated as time averaged maximum velocity multiplied by 0.62 , as previously reported $[7,8]$.

Portal blood flow (PBF) and hepatic artery blood flow (HABF) were obtained by multiplying the portal vein cross-sectional area, assuming a circular shape of the portal vein and hepatic artery section, by the mean velocity of blood flow in the vessel $[10,13$, 14] according to the following formula: 
Blood flow $(\mathrm{ml} / \mathrm{min})=$ cross sectional area of the vessel* mean flow velocity $(\mathrm{cm} / \mathrm{s}) * 60$ Total hepatic blood flow (US-HBF) was then calculated as PBF + HABF.

The percentage of US-HBF provided by $\mathrm{PBF}$ and $\mathrm{HABF}$ was calculated as: PBF/US$\mathrm{HBF}^{*} 100$ and $\mathrm{HABF} / \mathrm{US}-\mathrm{HBF}^{*} 100$.

Congestion index of the portal vein was calculated as previously reported by Moriyasu et al. [15] as follows:

Congestion Index $=$ cross-sectional area of the portal vein $\left(\mathrm{cm}^{2}\right) /$ portal vein mean flow velocity $(\mathrm{cm} / \mathrm{s})$.

\section{Statistical analysis}

Means of ICG-HBF and US-HBF were compared by paired T-test or Kruskall-Wallis test, while medians were compared by Wilcoxon's test. Correlations between ICG-HBF and US-HBF and its components were made by Pearson's test. Agreement between the two techniques was assessed by intraclass correlation coefficients (ICC) for consistency and absolute concordance. According to Landis et al. ICC were interpreted as follows: 0-0.2 indicates poor agreement: 0.3-0.4 indicates fair agreement; 0.5-0.6 indicates moderate agreement; 0.7-0.8 indicates strong agreement; and $>0.8$ indicates almost perfect agreement [16].

We arbitrarily defined as "clinically important difference" a difference between US$\mathrm{HBF}$ and $\mathrm{ICG}-\mathrm{HBF} \geq 20 \%$. Receiver operating characteristics curve (ROC) analysis was used to identify the most specific cut-off of the tested parameters able to detect this clinically important difference in HBF as compared with ICG-HBF.

The $\alpha$ value was set at 0.05 . All p-values are two-sided. Statistical analysis was performed with SPSS 16.0 package (SPSS, Chicago, IL, USA). 


\section{RESULTS}

Table 2 shows the results of DUS examination, US-HBF and ICG-HBF in the 50 patients included, and Table 3 shows the correlation of Doppler-US examination with HVPG, Child-Pugh score and grade of esophageal varices. As shown, among the Doppler-US variables studied, the congestion index of the portal vein significantly correlated with the HVPG and with the size of esophageal varices, but did not correlate with ICG-HBF $(\mathrm{R}=-0.047 ; \mathrm{p}=0.748)$.

Overall, PBF accounted for approximately $70 \%$ and $\mathrm{HABF}$ for $30 \%$ of $\mathrm{HBF}$, as estimated by DUS.

The absolute value of HBF was similar when measured by ICG and by DUS; being

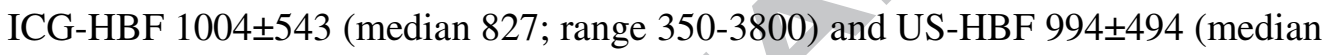
894; range 122-3088). The comparison revealed no statistically significant differences ( $\mathrm{p}=0.661$ for means; $\mathrm{p}=0.697$ for medians). However, despite mean and median values being similar, individual patients' data disclosed marked differences between both methods $(386 \pm 415 \mathrm{ml} / \mathrm{min})$. The discrepancy was "clinically significant" (>20\% of the total HBF) in 32 patients (64\%). Intraclass correlation coefficients between ICG-HBF and US-HBF were significant, but only fair: ICC consistency 0.456 (95\% CI 0.2000.654, $\mathrm{p}<0.0001$ ); ICC absolute agreement 0.461 (95\% CI 0.203-0.658, $\mathrm{p}<0.0001$ ). We next examined which factors could explain the discrepancy between the two methods, and found several factors that might have played a role. Firstly, the degree of discrepancy increased as HBF measured by any of the methods increased (KruskallWallis test: $\mathrm{p}=0.003$ ) (Figure 1 and 2A). Particularly outlier discrepancies were observed in patients in whom HBF by either method was over $1900 \mathrm{ml} / \mathrm{min}$, suggesting that values over this threshold should not be considered reliable. This occurred in 4 
patients in our series (8\%). Secondly, a significantly greater discrepancy between the two methods was observed in patients with ICG extraction $<0.20(n=17): 633 \pm 571$ vs. $274 \pm 261 \mathrm{ml} / \mathrm{min}(\mathrm{p}=0.004)$ in patients with $\mathrm{ICG}$ extraction $\geq 0.20(\mathrm{n}=33)$. Patients with low ICG extraction, as expected, had significantly more severe liver failure as compared with patients with higher ICG extraction (Child-Pugh score: $9.1 \pm 1.4$ vs. $6.0 \pm 1.4, \mathrm{p}<0.0001$; Albumin $29 \pm 3$ vs. $37 \pm 6 \mathrm{~g} / \mathrm{L}, \mathrm{p}<0.0001$; Bilirubin $3.1 \pm 1.1$ vs. $1.2 \pm 0.5 \mathrm{mg} / \mathrm{dl}, \mathrm{p}<0.0001$; INR $1.58 \pm 0.22$ vs. $1.21 \pm 0.16, \mathrm{p}<0.0001)$. In particular, two of the patients with ICG extraction $<0.20$ had extremely high, implausible values of $\mathrm{HBF}$ by ICG (namely $2552 \mathrm{ml} / \mathrm{min}$ and $3800 \mathrm{ml} / \mathrm{min}$ ), suggesting that gross overestimation by the gold-standard had probably occurred. Accordingly, we made an exploratory analysis restricted to patients with ICG extraction $\geq 0.20$. In this subgroup, the correlation between ICG-HBF and US-HBF improved significantly $(\mathrm{R}=0.651$, p<0.0001) (Figure 2B), and so did ICCs: consistency 0.616 (95\% CI 0.351-0.790, p<0.0001); ICC absolute agreement 0.602 (95\% CI 0.336-0.780, $\mathrm{p}<0.0001)$.

The magnitude of the discrepancy between ICG-HBF and US-HBF was also influenced by the degree of liver failure as estimated by the MELD score. Patients with MELD score $>10(n=28)$ had a significantly greater discrepancy than those with MELD $\leq 10$ $(490 \pm 482$ Vs $261 \pm 288 \mathrm{ml} / \mathrm{min} ; \mathrm{p}=0.048)$.

\section{Correlation between the components of US-HBF and ICG-HBF}

In the whole population we observed a significant correlation between PBF and ICGHBF $(\mathrm{R}=0.459, \mathrm{p}<0.001)$, while HABF and ICG-HBF were not significantly correlated $(\mathrm{R}=0.231, \mathrm{p}=0.114)$. This changed according to compensated or decompensated stage of the disease: in compensated patients the correlation between US-HBF and ICG-HBF 
$(\mathrm{R}=0.762, \mathrm{p}<0.0001)$ was entirely explained by the correlation of PBF and ICG-HBF $(\mathrm{R}=0.761, \mathrm{p}<0.0001)$, while in decompensated patients it was weaker $(\mathrm{R}=0.388$, $\mathrm{p}=0.050)$ and explained by $\operatorname{HABF}(\mathrm{R}=0.418, \mathrm{p}=0.034)$ but not by $\operatorname{PBF}(\mathrm{R}=0.185, \mathrm{NS})$.

We observed a direct correlation between the magnitude of discrepancy in HBF measurement between the two techniques and the proportion of US-HBF provided by HABF $(\mathrm{R}=0.371, \mathrm{p}=0.009)$, which was confirmed in patients with an optimal ICG-HBF measurement (those with ICG extraction above 0.20$)(\mathrm{R}=0.404, \mathrm{p}=0.018)$. This observation suggests that arterialization of the liver circulation reduces the accuracy of US-Doppler estimations of hepatic blood flow. On ROC curve analysis (Fig. 3), a cutoff percentage of $\mathrm{HABF} \geq 40 \%$ of total US-HBF had a $90 \%$ specificity to detect a discrepancy over $20 \%$ of US-HBF with ICG-HBF. Patients in whom the HABF was less than $40 \%$ of total US-HBF had a discrepancy between US-Doppler and ICG-HBF of $34 \pm 35 \%$ as compared with $65 \pm 43 \%$ in patients in whom the HAF accounted for over $40 \%$ of total $\mathrm{HBF}(\mathrm{p}=0.020)$. 


\section{DISCUSSION}

HBF measurement would be ideally needed to investigate the effects of new drugs potentially acting on portal hypertension, and to better stratify of the individual risk in patients with cirrhosis.

At first glance, the findings reported in the present paper suggest that ICG-HBF and US-HBF give similar results in patients with cirrhosis, as indicated by very close mean and median values. However, a closer look at the results shows that the two methods have substantial discrepancies in individual patients. We could identify a worse liver function (MELD score $>10$; ICG extraction <0.20), and a higher degree of arterialization of the liver circulation as factors increasing the difference of HBF results between the two methods. These factors may be used in practice to select the patients that can be confidently studied non-invasively by Doppler US. Our results provide evidence that Doppler ultrasound measurement of $\mathrm{HBF}$, calculated as the sum of portal blood flow and hepatic artery blood flow, might be used as a reasonable non-invasive surrogate of the measurement obtained by the invasive gold-standard technique (ICG infusion-based estimation) in those patients with cirrhosis and portal hypertension and with normal hepatic artery blood flow (HABF by US-Doppler below $35 \%$ of total US$\mathrm{HBF}$ ), while patients with a higher arterial component of total hepatic blood flow, as it occurs in patients with advanced portal hypertension and extensive portal-systemic collaterals, its results should instead be considered insufficiently reliable.

As any diagnostic technique, the estimation of HBF by Doppler ultrasound has advantages and limitations. A clear benefit over ICG-based method is given by the lack of invasiveness, by obtaining results in real time, and by the possibility of evaluating separately arterial and portal venous supply. In vitro studies demonstrated that US-HBF 
is accurate and reproducible $[9,17]$, and age, sex, height, weight and body surface area do not modify the accuracy of measurements of PBF by DUS in human subjects [18]. On the other hand it is well known that Doppler measurements in vivo exhibit a large variability which can be due to anatomical (limitations of the angle between the Doppler beam and the vessel), physiologic phenomena (e.g. meal ingestion) and to intra- and inter-observer errors $[19,20]$. While anatomical features cannot be modified, variability due to the remaining factors can be minimized by applying standardized protocols of examination as suggested by EFSUMB recommendations [8], which were carefully adhered to in the present study.

Despite using a careful, standardized protocol for DUS examination [8] we observed that the discrepancy between US-HBF (and PBF) by DUS and ICG-HBF increased as the value of HBF by any of the two methods increased. This data is in agreement with the results obtained by Bolognesi et al. in 27 patients with cirrhosis regarding the agreement of PBF by DUS and ICG-HBF [11]. In contrast, we could not reproduce the existence of a strong correlation between PBF by DUS and ICG-HBF in the whole population we included. This probably depends upon differences in the proportion of patients with compensated and decompensated cirrhosis included in the two studies, since at a closer look of our data US-HBF and PBF by DUS were well correlated with ICG-HBF in patients with well compensated cirrhosis, who were the majority in the study by Bolognesi et al [11]. Another major difference with the study by Bolognesi et al. [11] regards the availability of data on $\mathrm{HABF}$ in our study.

Our data suggest that while $\mathrm{HABF}$ is minimally relevant in terms of correlation with ICG-HBF in patients with compensated cirrhosis and preserved liver function, its 
careful estimation is very important in patients with decompensated cirrhosis and advanced liver failure, since in this subset no correlation between PBF by DUS and ICG-HBF exists. This supports that hepatic blood flow is largely influenced by HABF rather than by $\mathrm{PBF}$ in decompensated patients, who very frequently have extensive portal-systemic collaterals. This is well in accordance with the fact that, as demonstrated by our study, the more arterialized the liver is, the less reliable is the measurement of HBF by Doppler, as compared to the gold-standard technique.

Some limitations of our work should be acknowledged. The first is due to the fact that HBF estimation by ICG technique is an imperfect gold-standard. The rational for using this method is the fact that ICG is primarily non-reversibly extracted by the liver (about 70\%) [21], being HBF the main determinant of its clearance. Nonetheless, it has been previously shown that errors in the estimation of HBF by ICG arise when the hepatic extraction of the compound is $<10 \%$, as it may occur in patients with severe hepatic failure. In this paper we excluded this population, and restricted the analysis to patients with ICG extraction $>0.1$; still, two patients with ICG extraction index below 0.2 had very high, unlikely values of ICG-HBF, suggesting an estimation error by this technique. New methods of liver perfusion estimation, such as the application of mathematical models (e.g. dual-inlet two-compartment uptake model) to dynamic contrast-enhanced (DCE) magnetic resonance imaging (MRI) using a specific hepatobiliary contrast agent (gadoxetic acid) [22], or positron emission tomography (PET) using oxygen-15 labeled water [23], might represent better gold-standards for future studies, although may be inconvenient for its use in clinical practice. 
Another limitation is due to our inclusion criteria, which limited the study to patients in whom both PBF and HABF could be measured. It was previously reported that hepatic artery visualization is possible only in a minority of patients with cirrhosis $[9,11]$; this was not the case in our series, since both the portal vein and the hepatic artery could be assessed in $85 \%$ of cases. Advances in the technology applied to ultrasound equipments might explain this discrepancy.

Since only one physician performed all the measurements, interobserver variability was not assessed in the present study. However, as previously stated, we applied standardized, well accepted protocols of examination [8] which have been previously shown to reduce interobserver variability $[19,20]$.

Finally, in order to give a pragmatic applicability of our findings, we examined which factor may allow excluding patients with an excessive risk of unreliable results on the basis of Doppler measurements of total hepatic blood flow, that we arbitrarily set as difference of $>20 \%$ ("clinically relevant difference"). In that regard, it is important to remark our finding that patients with excessive liver arterialization (i.e., a hepatic artery fraction of total liver blood flow $>40 \%$ ) had a $90 \%$ chance of exhibiting such a clinically significant discrepancy and therefore, US-HBF should be considered unreliable in such patients. Even if we believe that this finding is reasonably robust, we acknowledge that it can be questioned until confirmed by independent studies.

In conclusion, our data provide new evidence regarding the use of Doppler-US as a noninvasive method to estimate hepatic blood flow. Future studies using the combination of Doppler-US and elastographic methods [24-26] for non-invasive assessment of changes 
in intrahepatic haemodynamics (a new target for the treatment of portal hypertension [27]) should take into account the limitations of Doppler-US.

\section{ACKNOWLEDGMENTS}

The authors are indebted to Ms. M.A. Baringo, L. Rocabert, R. Saez and Lara Orts for their expert nursing assistance. The authors also express their gratitude to Ms. Clara Esteva for her secretarial support. 
Table 1. Main clinical, laboratory and hemodynamic features of the studied population $(n=50)$.

\begin{tabular}{|c|c|}
\hline Characteristic & \\
\hline Age (yrs) & $56 \pm 9$ \\
\hline Gender, n (M/F) & $33 / 17$ \\
\hline Body surface area $\left(\mathrm{m}^{2}\right)$ & $1.81 \pm 0.16$ \\
\hline $\begin{array}{l}\text { Etiology, } \mathrm{n} \\
\text { (HCV/HBV/alcohol/other) }\end{array}$ & $25 / 2 / 15 / 8$ \\
\hline Child-Pugh class $(\mathrm{A} / \mathrm{B} / \mathrm{C})$ & $26 / 13 / 11$ \\
\hline Child-Pugh score & $7.0 \pm 2.2$ \\
\hline $\begin{array}{l}\text { Esoph. Varices } \\
\text { (no/small/large) }\end{array}$ & $12 / 15 / 23$ \\
\hline MELD score & $11 \pm 4$ \\
\hline Treatment with beta-blockers, $\mathrm{n}(\%)$ & $13(26 \%)$ \\
\hline Ascites, n (\%) & $18(36 \%)$ \\
\hline Previous decompensation, n (\%) & $22(44 \%)$ \\
\hline Bilirubin (mg/dl) & $1.8 \pm 1.4$ \\
\hline INR & $1.29 \pm 0.25$ \\
\hline Albumin (g/dl) & $3.6 \pm 0.7$ \\
\hline Creatinine $(\mathrm{mg} / \mathrm{dl})$ & $0.98 \pm 0.23$ \\
\hline Platelets $\left(\mathrm{n}^{3} / \mathrm{mmc}\right)$ & $115 \pm 63$ \\
\hline Spleen diameter $(\mathrm{cm})$ & $14.9 \pm 2.8$ \\
\hline HVPG $(\mathrm{mmHg})$ & $16.6 \pm 6.0$ \\
\hline ICG hepatic clearance $(\mathrm{ml} / \mathrm{min})$ & $208 \pm 127$ \\
\hline ICG extraction index (\%) & $38 \pm 23$ \\
\hline Mean arterial pressure $(\mathrm{mmHg})$ & $90 \pm 14$ \\
\hline Heart rate (beats per minute) & $74 \pm 15$ \\
\hline
\end{tabular}


Table 2. Results of DUS examination and ICG-HBF in the studied population. Data are given overall and according to Child-Pugh classes as mean \pm standard deviation.

\begin{tabular}{|c|c|c|c|c|c|}
\hline \multicolumn{2}{|c|}{ Parameter measured } & \multirow{2}{*}{$\begin{array}{c}\text { Overall }(n=50) \\
12.4 \pm 2.4\end{array}$} & \multirow{2}{*}{$\begin{array}{l}\text { Child A } \\
(n=26)\end{array}$} & \multirow{2}{*}{$\begin{array}{l}\text { Child B } \\
(\mathrm{n}=13) \\
13.8 \pm 1.4\end{array}$} & \multirow{2}{*}{$\begin{array}{c}\begin{array}{c}\text { Child C } \\
(\mathrm{n}=11)\end{array} \\
11.7 \pm 1.7\end{array}$} \\
\hline Portal & Diameter (mm) & & & & \\
\hline & $\begin{array}{l}\text { Time averaged } \\
\text { maximal velocity } \\
(\mathrm{cm} / \mathrm{sec})\end{array}$ & $16.5 \pm 5.6$ & $17.0 \pm 5.0$ & $17.5 \pm 6.2$ & $14.2 \pm 5.9$ \\
\hline & $\begin{array}{l}\text { Volume of flow } \\
(\mathrm{ml} / \mathrm{min})\end{array}$ & $715 \pm 349$ & $685 \pm 309$ & $895 \pm 383$ & $574 \pm 340$ \\
\hline & Congestion index & $0.14 \pm 0.06$ & $0.13 \pm 0.07$ & $0.16 \pm 0.07$ & $0.14 \pm 0.04$ \\
\hline & $\begin{array}{l}\% \text { of total blood } \\
\text { flow }\end{array}$ & $72.6 \pm 16.8$ & $71.4 \pm 15.6$ & $77.9 \pm 18.5$ & $68.5 \pm 17.4$ \\
\hline \multirow{4}{*}{$\begin{array}{l}\text { Hepatic } \\
\text { artery }\end{array}$} & Diameter (mm) & $4.0 \pm 1.2$ & $4.2 \pm 1.3$ & $3.9 \pm 1.3$ & $3.6 \pm 0.5$ \\
\hline & $\begin{array}{l}\text { Time averaged } \\
\text { maximal velocity } \\
(\mathrm{cm} / \mathrm{sec})\end{array}$ & $36.3 \pm 19.3$ & $34.8 \pm 12.7$ & $39.4 \pm 31.3$ & $36.3 \pm 15.3$ \\
\hline & $\begin{array}{l}\text { Volume of flow } \\
\text { (ml/min) }\end{array}$ & $274 \pm 286$ & $288 \pm 320$ & $283 \pm 319$ & $225 \pm 127$ \\
\hline & $\begin{array}{l}\% \text { of total blood } \\
\text { flow }\end{array}$ & $27.4 \pm 16.8$ & $28.6 \pm 15.6$ & $22.1 \pm 18.6$ & $31.5 \pm 17.4$ \\
\hline $\begin{array}{l}\text { US-HBF } \\
(\mathrm{ml} / \mathrm{min})\end{array}$ & & $994 \pm 494$ & $973 \pm 533$ & $1177 \pm 448$ & $807 \pm 402$ \\
\hline $\begin{array}{l}\text { ICG-HB } \\
(\mathrm{ml} / \mathrm{min})\end{array}$ & & $1004 \pm 543$ & $843 \pm 345$ & $1212 \pm 672$ & $1147 \pm 344$ \\
\hline
\end{tabular}


Table 3. Correlation of US findings and ICG-HBF with HVPG, Child-Pugh score and grade of esophageal varices. $\mathrm{P}$ values in bold are statistically significant $(<0.05)$.

\begin{tabular}{|c|c|c|c|c|c|c|c|}
\hline & & \begin{tabular}{|c|} 
Correlation \\
with \\
HVPG \\
(R)
\end{tabular} & $\mathrm{p}$ & \begin{tabular}{|c|} 
Correlation \\
with \\
Child- \\
Pugh score \\
(R)
\end{tabular} & $\mathrm{p}$ & $\begin{array}{c}\text { Correlati } \\
\text { on with } \\
\text { EV } \\
\text { (no/Small } \\
\text { /Large) } \\
\text { (R) }\end{array}$ & $\mathrm{p}$ \\
\hline \multirow{5}{*}{$\begin{array}{l}\text { Portal } \\
\text { vein }\end{array}$} & Diameter (mm) & 0.111 & 0.419 & -0.026 & 0.851 & 0.574 & $<0.001$ \\
\hline & $\begin{array}{l}\text { Time averaged } \\
\text { maximal velocity } \\
(\mathrm{cm} / \mathrm{sec}) \\
\end{array}$ & -0.230 & 0.091 & -0.260 & \begin{tabular}{|l|}
0.049 \\
\end{tabular} & -0.012 & 0.932 \\
\hline & $\begin{array}{l}\text { Volume of flow } \\
\text { (ml/min) }\end{array}$ & -0.040 & 0.769 & -0.139 & 0.312 & 0.350 & 0.009 \\
\hline & Congestion index & 0.296 & 0.028 & 0.139 & 0.311 & 0.466 & $<0.001$ \\
\hline & $\begin{array}{l}\% \text { of total blood } \\
\text { flow }\end{array}$ & -0.111 & 0.430 & -0.137 & 0.327 & -0.272 & 0.049 \\
\hline \multirow{4}{*}{$\begin{array}{l}\text { Hepatic } \\
\text { artery }\end{array}$} & Diameter (mm) & 0.075 & 0.592 & -0.073 & 0.602 & 0.204 & 0.143 \\
\hline & $\begin{array}{l}\text { Time averaged } \\
\text { maximal velocity } \\
(\mathrm{cm} / \mathrm{sec})\end{array}$ & 0.040 & 0.769 & 0.128 & 0.352 & 0.078 & 0.572 \\
\hline & $\begin{array}{l}\text { Volume of flow } \\
\text { (ml/min) }\end{array}$ & 0.057 & 0.685 & 0.006 & 0.964 & 0.102 & 0.467 \\
\hline & $\begin{array}{l}\% \text { of total blood } \\
\text { flow }\end{array}$ & 0.111 & 0.430 & 0.137 & 0.327 & 0.272 & 0.049 \\
\hline \multicolumn{2}{|l|}{$\begin{array}{l}\text { US-HBF } \\
(\mathrm{ml} / \mathrm{min})\end{array}$} & -0.005 & 0.970 & -0.105 & 0.454 & 0.355 & 0.009 \\
\hline \multicolumn{2}{|c|}{$\begin{array}{l}\text { ICG-HBF } \\
(\mathrm{ml} / \mathrm{min})\end{array}$} & 0.013 & 0.930 & 0.113 & 0.436 & 0.222 & 0.122 \\
\hline \multicolumn{2}{|c|}{ HVPG (mmHg) } & --- & --- & 0.577 & $<0.001$ & 0.249 & 0.021 \\
\hline
\end{tabular}




\section{Figure legends}

Figure 1. Magnitude of the difference ( $\mathrm{ml} / \mathrm{min}$ ) observed between US-HBF and ICGHBF according to the highest HBF as measured by any of the two methods.

Figure 2. Correlation between US-HBF and ICG-HBF in the studied patients. Panel ACorrelation between US-HBF and ICG-HBF in the whole population of the study $(\mathrm{n}=50 ; \mathrm{R}=0.464 ; \mathrm{p}<0.0001)$. Panel B-Correlation between US-HBF and ICG-HBF in patients with ICG extraction $>0.20(\mathrm{n}=33 ; \mathrm{R}=0.651 ; \mathrm{p}<0.0001)$.

Figure 3. ROC curve plotting the HABF percentage of US-HBF to discriminate patients with discrepancies between US-HBF and ICG-HBF $\geq 20 \%$ (AUROC $0.711 ; 95 \%$ CI $0.533-0.892 ; \mathrm{p}=0.043)$. The circle identifies the $90 \%$ specific cut-off chosen, consisting in a HABF component of over $40 \%$ of US-HBF. 


\section{References}

[1] Garcia-Tsao G, Bosch J. Management of varices and variceal hemorrhage in cirrhosis. N Engl J Med 2010;362:823-832.

[2] Bosch J, Abraldes JG, Berzigotti A, Garcia-Pagan JC. The clinical use of HVPG measurements in chronic liver disease. Nat Rev Gastroenterol Hepatol 2009;6:573-582.

[3] Clemmesen JO, Tygstrup N, Ott P. Hepatic plasma flow estimated according to Fick's principle in patients with hepatic encephalopathy: evaluation of indocyanine green and Dsorbitol as test substances. Hepatology 1998;27:666-673.

[4] Bradley SE. Estimation of hepatic blood flow. Methods Med Res 1960;8:275-283.

[5] Navasa M, Bosch J, Mastai R, et al. Measurement of hepatic blood flow, hepatic extraction and intrinsic clearance of indocyanine green in cirrhosis. Comparison of a non-invasive pharmacokinetic method with measurements using hepatic vein catheterization. Eur $\mathbf{J}$ Gastroenterol Hepatol 1991;3:305-312.

[6] Navasa M, Garcia-Pagan JC, Bosch J, Rodes J. [Prognostic value of hepatic clearance of indocyanine green in patients with liver cirrhosis and hemorrhage of esophageal varices]. Med Clin (Barc ) 1992;98:290-294.

[7] Berzigotti A, Piscaglia F. Ultrasound in portal hypertension--part 1. Ultraschall Med 2011;32:548-568.

[8] Berzigotti A, Piscaglia F. Ultrasound in portal hypertension--part 2--and EFSUMB recommendations for the performance and reporting of ultrasound examinations in portal hypertension. Ultraschall Med 2012;33:8-32.

[9] Carlisle KM, Halliwell M, Read AE, Wells PN. Estimation of total hepatic blood flow by duplex ultrasound. Gut 1992;33:92-97.

[10] Moriyasu F, Ban N, Nishida O, Nakamura T, Miyake T, Uchino H, et al. Clinical application of an ultrasonic duplex system in the quantitative measurement of portal blood flow. J Clin Ultrasound 1986;14:579-588.

[11] Bolognesi M, Sacerdoti D, Merkel C, Gatta A. Relationship between portal blood flow measured by image-directed Doppler ultrasonography and hepatic blood flow measured by indocyanine green constant infusion in patients with cirrhosis. J Clin Ultrasound 1995;23:297-303.

[12] Navasa M, Chesta J, Bosch J, Rodes J. Reduction of portal pressure by isosorbide-5mononitrate in patients with cirrhosis. Effects on splanchnic and systemic hemodynamics and liver function. Gastroenterology 1989;96:1110-1118.

[13] Bolognesi M, Sacerdoti D, Merkel C, Gerunda G, Maffei-Faccioli A, Angeli P, et al. Splenic Doppler impedance indices: influence of different portal hemodynamic conditions [see comments]. Hepatology 1996;23:1035-1040. 
[14] Sabba C, Ferraioli G, Sarin SK, Lerner E, Groszmann RJ, Taylor KJ. Feasibility spectrum for Doppler flowmetry of splanchnic vessels. In normal and cirrhotic populations. J Ultrasound Med 1990;9:705-710.

[15] Moriyasu F, Nishida O, Ban N, Nakamura T, Sakai M, Miyake T, et al. "Congestion index" of the portal vein. AJR Am J Roentgenol 1986;146:735-739.

[16] Landis JR, Koch GG. The measurement of observer agreement for categorical data. Biometrics 1977;33:159-174.

[17] Nakamura T, Moriyasu F, Ban N, Nishida O, Tamada T, Kawasaki T, et al. Quantitative measurement of abdominal arterial blood flow using image-directed Doppler ultrasonography: superior mesenteric, splenic, and common hepatic arterial blood flow in normal adults. J Clin Ultrasound 1989;17:261-268.

[18] de Vries PJ, van HJ, Hoekstra JB, de HP. Duplex Doppler measurements of portal venous flow in normal subjects. Inter- and intra-observer variability. J Hepatol 1991;13:358-363.

[19] Sabba C, Merkel C, Zoli M, Ferraioli G, Gaiani S, Sacerdoti D, et al. Interobserver and interequipment variability of echo-Doppler examination of the portal vein: effect of a cooperative training program. Hepatology 1995;21:428-433.

[20] Sacerdoti D, Gaiani S, Buonamico P, Merkel C, Zoli M, Bolondi L, et al. Interobserver and interequipment variability of hepatic, splenic, and renal arterial Doppler resistance indices in normal subjects and patients with cirrhosis. J Hepatol 1997;27:986-992.

[21] Paumgartner G. The handling of indocyanine green by the liver. Schweiz Med Wochenschr 1975;105:1-30.

[22] Sourbron S, Sommer WH, Reiser MF, Zech CJ. Combined quantification of liver perfusion and function with dynamic gadoxetic acid-enhanced MR imaging. Radiology 2012;263:874883.

[23] Ziegler SI, Haberkorn U, Byrne H, Tong C, Kaja S, Richolt JA, et al. Measurement of liver blood flow using oxygen-15 labelled water and dynamic positron emission tomography: limitations of model description. Eur J Nucl Med 1996;23:169-177.

[24] Berzigotti A, Seijo S, Arena U, Abraldes JG, Vizzutti F, Garcia-Pagan JC, et al. Elastography, spleen size, and platelet count identify portal hypertension in patients with compensated cirrhosis. Gastroenterology 2013;144:102-111.

[25] Takuma Y, Nouso K, Morimoto Y, Tomokuni J, Sahara A, Toshikuni N, et al. Measurement of spleen stiffness by acoustic radiation force impulse imaging identifies cirrhotic patients with esophageal varices. Gastroenterology 2013;144:92-101.

[26] Colecchia A, Montrone L, Scaioli E, Bacchi-Reggiani ML, Colli A, Casazza G, et al. Measurement of Spleen Stiffness to Evaluate Portal Hypertension and the Presence of Esophageal Varices in Patients With HCV-Related Cirrhosis. Gastroenterology 2012;143:646-654. 
[27] Garcia-Pagan JC, Gracia-Sancho J, Bosch J. Functional aspects on the pathophysiology of portal hypertension in cirrhosis. J Hepatol 2012;57:458-461. 


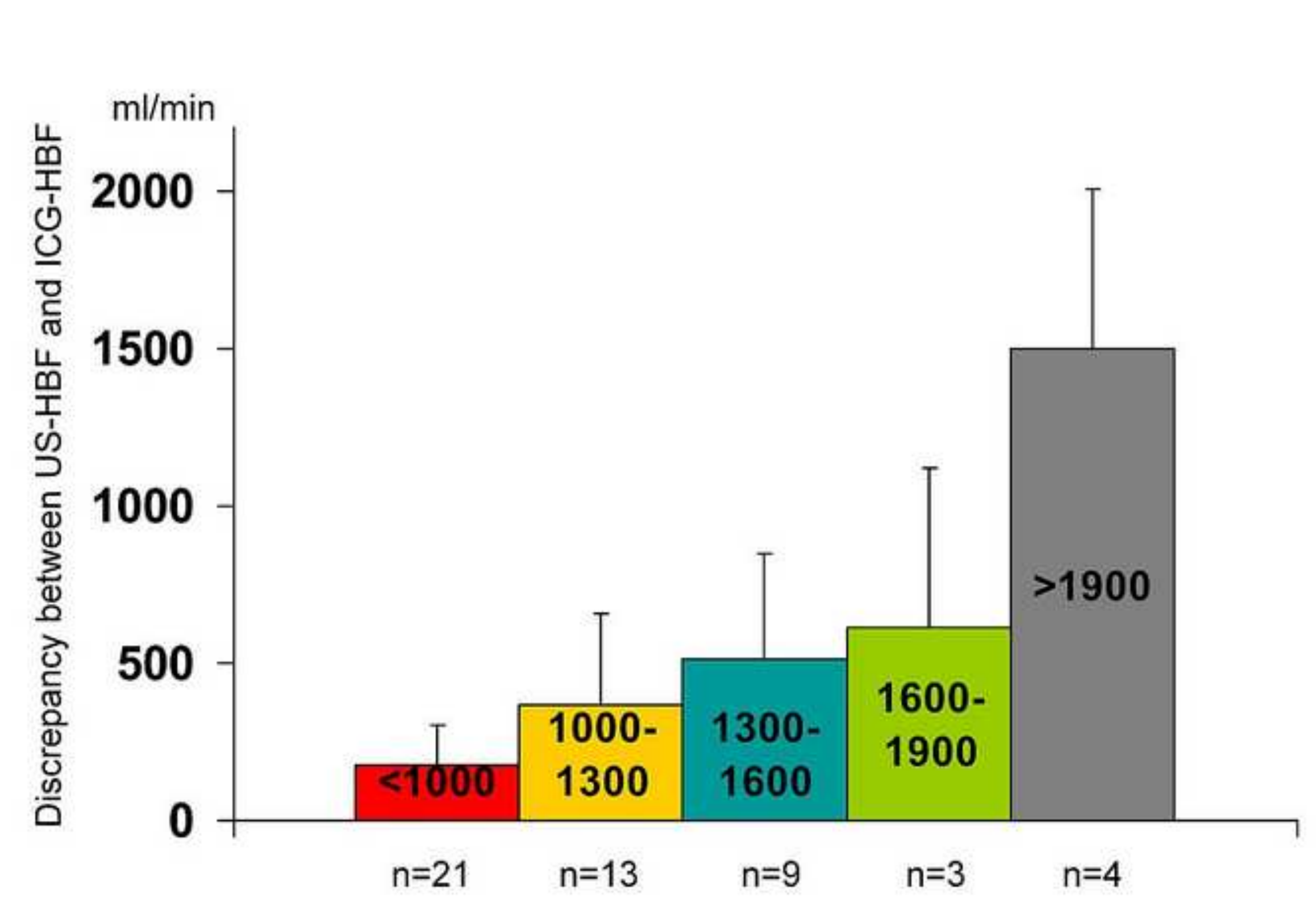

.

(
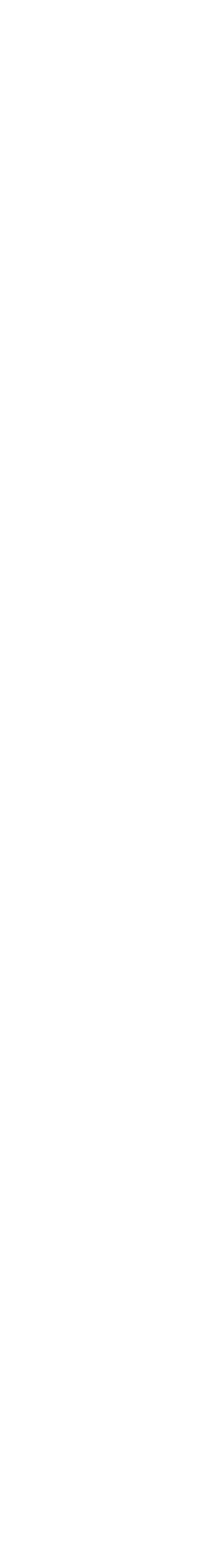
Figure 2

ACCEPTED MANUSCRIPT
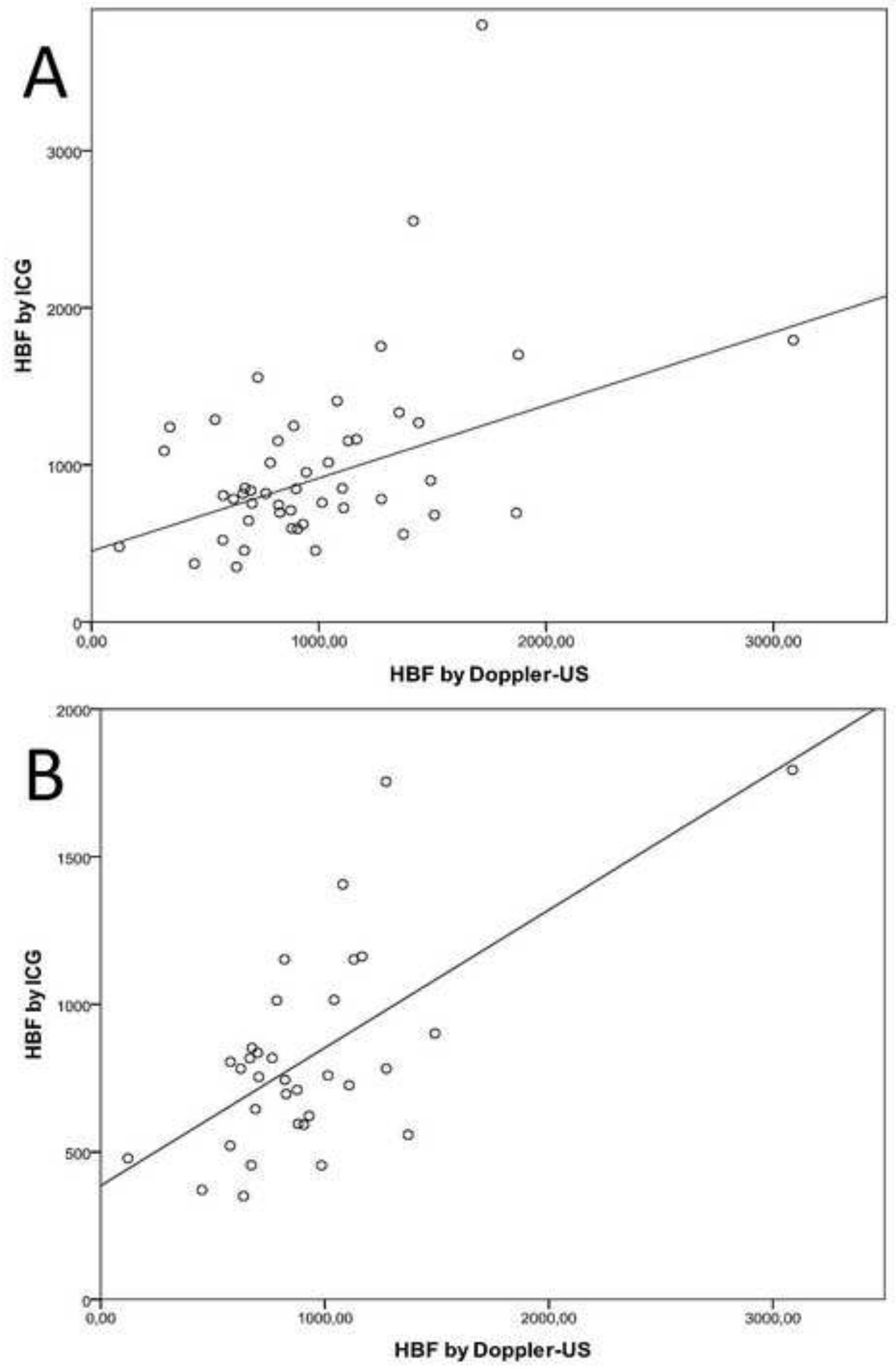
ACCEPTED MANUSCRIPT

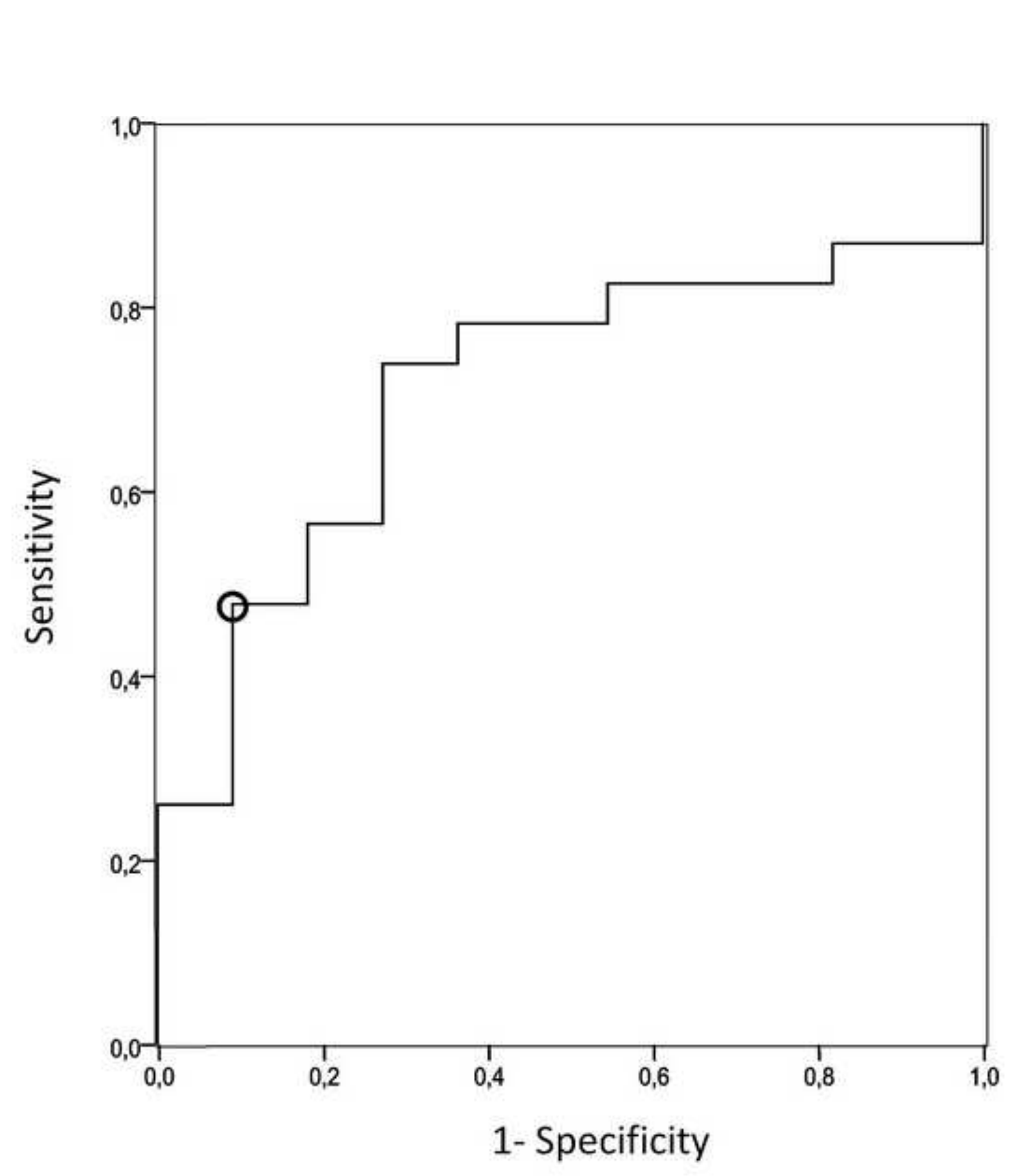

Figure 3

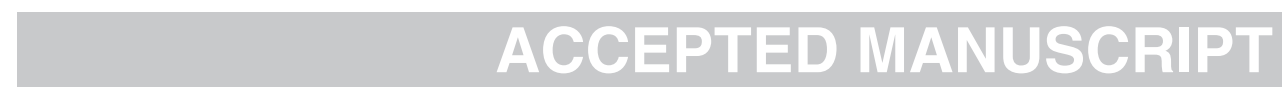

.

